\title{
Valproate and childbearing potential: new regulations
}

\section{Sanjay M Sisodiya, and Epilepsy Advisory Group for the Association of British Neurologists}

Department of Clinical and Experimental Epilepsy, UCL Institute of Neurology, London, UK

\section{Correspondence to}

Professor Sanjay M Sisodiya, Department of Clinical and Experimental, UCL Institute of Neurology, London WC1N 3BG, UK; s.sisodiya@ucl.ac.uk

Accepted 20 March 2018 Published Online First 23 April 2018

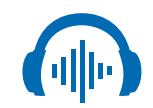

Listen to Podcast www.goo.gl/sNwjuh

\section{Sinked}

http://dx.doi.org/10.1136/ practneurol-2018-001932

\section{Check for updates}

To cite: Sisodiya SM, and Epilepsy Advisory Group for the Association of British Neurologists. Pract Neurol 2018;18:176-178.
Each encounter between a patient and clinician is likely to include an explicit or implicit evaluation of benefit versus risk and should always be an exercise in person-centred medicine. New stipulations from the Coordination Group for Mutual Recognition and Decentralised Procedures-Human (CMDh), a regulatory body representing European Union member states, about the use of valproate in women of childbearing potential ${ }^{1}$ bring these age-old considerations into sharp relief. There will be a change in the marketing authorisation: valproate will be contraindicated in pregnancy and women of childbearing potential not using effective contraception. This requires all clinicians who see people with epilepsy to think carefully again, even before changes to UK use of valproate are enforced by changes in the licence from the UK Medicines and Healthcare products Regulatory Agency (MHRA). The many challenges are brought out in two accompanying articles in this issue. ${ }^{23}$ Importantly, no one should stop valproate use abruptly because of this announcement, without consulting their doctor as doing so could result in harm to themselves or to an unborn child.

The background is well established and largely derived from prospective registries. ${ }^{4}$ Babies born to mothers who take valproate medicines during pregnancy have up to a $10 \%$ risk of birth defects, compared with 2\%-3\% background risk, ${ }^{5}$ and a variable, sometimes unknown, risk on polytherapy with other antiepileptic drugs. ${ }^{6}$ Some of these malformations may necessitate early corrective surgery; some are inoperable. There is an average reduction in IQ estimated at 6-11 points ${ }^{7}$ 8 and $30 \%-40 \%$ risk of developmental disability. ${ }^{9-11}$ Compared with the general population, the risk of autism spectrum disorders is threefold and that of attention-deficit hyperactivity disorder is fivefold. ${ }^{12-14}$ These consequences may come with a lifetime burden of medical and social care for the family and the state. While some structural abnormalities may be detectable on prenatal screening, the intellectual and behavioural outcomes cannot be predicted. Animal models suggest that some risks may be passed to unexposed generations epigenetically ${ }^{15}$ : this work needs replication and there are no studies in humans. Whatever additional risks emerge, there are already significant known risks associated with the use of valproate in pregnancy. We should not assume any disease specificity: any use of valproate for any condition should be considered to carry these risks.

In response to the accumulated evidence on risks, the MHRA issued advice to prescribers in 2015 and again in 2016 (https://www.gov.uk/drug-safety-update/ medicines-related-to-valproate-risk-ofabnormal-pregnancy-outcomes). Materials to communicate the risks included a booklet for professionals, a consultation checklist and a guide and card for patients.

However, patient surveys suggested that the message had not got through: about one in five women of childbearing potential remained unaware of the risks (https://www.epilepsysociety. org.uk/news/Women-not-aware-epilepsymedicine-risk-pregnancy-27-09-2017). Following a further review and public hearing in London by the European Pharmacovigilance Risk Assessment Committee (PRAC), further guidance was issued by the PRAC and subsequently now by CMDh. These measures will strengthen restrictions on valproate use and introduce new measures to require appropriate counselling and information for affected women. A mandatory pregnancy prevention programme will be introduced, supported by a revised 
Box 1 The main points of Coordination Group for Mutual Recognition and Decentralised ProceduresHuman (CMDh) measures on valproate use in women of childbearing potential and the pregnancy prevention programme

- Valproate should never be started unless alternative treatments are not suitable.

- In pregnancy, valproate must not be used. However, it is recognised that for some women with epilepsy, it may not be possible to stop valproate and they may have to continue treatment (with appropriate specialist care) in pregnancy.

- In female patients able to have children, valproate must not be used unless the conditions of the new pregnancy prevention programme are met.

- There will be changes to the product information reflecting the new conditions.

- Outer packaging of all valproate medicines must include a visual warning about the risks in pregnancy. In addition to boxed text, this may include a symbol/ pictogram, with the details to be adapted at national level.

- A patient reminder card will also be attached to the outer package for pharmacists to discuss with the patient each time the medicine is dispensed.

- Companies that market valproate should also provide updated educational materials in the form of guides for healthcare professionals and patients.

- The main points of the new valproate pregnancy prevention programme are:

- Assessing patients for the potential of becoming pregnant

- Pregnancy tests before starting and during treatment as needed

- Counselling patients about the risks of valproate treatment

- Explaining the need for effective contraception throughout treatment

- Carrying out reviews of treatment by a specialist at least annually

- Introduction of a new risk acknowledgement form that patients and prescribers will go through at each such review to confirm that appropriate advice has been given and understood.

- It is important that no woman should stop taking sodium valproate without first consulting her doctor. Sodium valproate is available in the UK under brand names such as Epilim, Epival, Episenta, Convulex and Orlept.

toolkit, with signed acknowledgement required. Box 1 lists the main points in the regulations.

There are important points to be brought out. The simplest option might be to avoid valproate completely in women of childbearing potential. But, based on one of the few randomised controlled trials in epilepsy, valproate is the best drug for some types of epilepsy. ${ }^{16}$ Valproate use has not been banned in girls and women of childbearing potential. We must guard against inadvertently replacing the problems of teratogenicity and neurotoxicity with that of an increased risk of sudden unexpected death in epilepsy because of loss of seizure control. The new regulations note: 'there may be a small number of women with epilepsy for whom there is no suitable alternative treatment to valproate and who should be appropriately supported and counselled.'.

The responsibility is on prescribers to facilitate informed consultation with women for whom valproate is being considered. The continued use of valproate requires proper informed discussion. Valproate may be the only effective drug for some people and others will be faced by the choice between the known risks of valproate and unknown risks of newer antiepileptic drugs. The regulations state the need for: 'an assessment of each patient's potential for becoming pregnant'. For example, some women cannot have children for medical reasons and this will apply to some with severe epilepsies for which valproate is a key element of treatment; some people choose not to have children. So the discussion between clinicians and women with epilepsy needs to be appropriate and individualised. Like all people with epilepsy, women of childbearing potential taking valproate need at least annual review. These are important and sensible regulations, and it is vital that all prescribers, in primary and other care, and dispensers are made aware and heed them. The particular concern about counselling women who may not have had a consultation with an epilepsy specialist for some time, particularly those who have well-controlled epilepsy, may have been started on valproate before puberty, and are seen only in primary care, will be covered by the new regulations.

Valproate can be a good drug for seizure control, and for some is life-saving, but comes with significant risks. The new regulations aim to reduce the number of people of childbearing potential taking valproate. The effects of changing patterns of prescribing and reduced use of valproate need monitoring. There will probably be a registry of women who remain on valproate: such monitoring should also be extended to women who have come off valproate, so that the full effects of the new regulations can be evaluated at individual and population-wide levels. There remain challenges and opportunities: responsibilities and resources will both need allocation. If patients are empowered to make their decisions, there are likely to be some women who, even after informed consultation, choose to carry a pregnancy while taking valproate. There may be dose-dependence for some consequences, ${ }^{8}$ and if valproate is used, dose reduction to the smallest effective dose, in two or three divided doses, should be considered, along with $5 \mathrm{mg}$ folic acid daily (these are not part of CMDh measures). ${ }^{8}$ Discussion with 
support groups may help, and alternative childbearing options may be relevant. It would not be appropriate to admonish either prescribers who accommodate this option or the women themselves, but this decision must be well documented and should be periodically revisited.

There will never be a trial of valproate and pregnancy outcomes in women of childbearing potential; so it is more important than ever that historical observational data are collected systematically and published. Fundamental research into the mechanisms of action and toxicity of valproate and possible genetic or other biomarkers of toxicity is essential, because as far as we can tell from the current data, some exposed children are not affected. But unless and until rational and effective selection of valproate as a credibly safe therapy for some individual women becomes a reality, we should follow the new regulations. These regulations aim to increase safety, and where their application may be complex, epilepsy specialists should be involved. We need to manage and minimise risk.

Collaborators H Angus-Leppan, SH Eriksson, AG Marson, R Mohanraj, N Moran, R Powell, C Price, G Warner, S Wroe.

Funding The authors have not declared a specific grant for this research from any funding agency in the public, commercial or not-for-profit sectors.

Competing interests HAL reports honoraria and a grant for investigator-initiated research (non-pharmacological) from Eisai; SHE reports honoraria/travel support from UCB and Eisai; AGM is a member of the steering committee of the EpiPGX consortium and has nothing else to report; RM reports honoraria/conference hospitality from UCB, Eisai and Livanova; NM has nothing to report; RP reports honoraria from UCB, Eisai and Janssen; CP reports travel bursaries from Biogen and UCB; GW has nothing to report; SW has nothing to report; SMS reports representing the Association of British Neurologists and The Royal College of Physicians (London) at the MHRA Valproate Stakeholders Network, is the Chief Investigator of the EpiPGX consortium (which has undertaken research on comparative effectiveness of drugs including valproate in juvenile myoclonic epilepsy, and on genetic variants for valproate-related teratogenicity), is a member of the scientific advisory board of Dravet Syndrome UK, patron of AHC UK, and Honorary Consultant Neurologist and Director of Genomics at the charity Epilepsy Society, and has received honoraria or grant funding from UCB, Eisai, Vitaflo and Nutricia. None of these affect the current article.

Patient consent Not required.

Provenance and peer review Commissioned; internally peer reviewed.

(c) Article author(s) (or their employer(s) unless otherwise stated in the text of the article) 2018. All rights reserved. No commercial use is permitted unless otherwise expressly granted.

\section{REFERENCES}

1 New measures to avoid valproate exposure in pregnancy endorsed. http://www.ema.europa.eu/ema/index.jsp?curl= pages/news_and_events/news/2018/03/news_detail_002929. jsp\&mid =WC0b01ac058004d5c1.

2 Lawthom C. Valproate and epilepsy: for women as well as men. Pract Neurol 2018;18:222-3.

3 Craig J. Women and Valproate: what should neurologists do? Pract Neurol 2018;18:219-21.

4 Bromley RL, Calderbank R, Cheyne CP, et al. Cognition in school-age children exposed to levetiracetam, topiramate, or sodium valproate. Neurology 2016;87:1943-53.

5 Weston J, Bromley R, Jackson CF, et al. Monotherapy treatment of epilepsy in pregnancy: congenital malformation outcomes in the child. Cochrane Database Syst Rev 2016;11:CD010224.

6 Vajda FJ, O'Brien TJ, Lander CM, et al. Antiepileptic drug combinations not involving valproate and the risk of fetal malformations. Epilepsia 2016;57:1048-52.

7 Meador KJ, Baker GA, Browning N, et al. Cognitive function at 3 years of age after fetal exposure to antiepileptic drugs. $N$ Engl J Med 2009;360:1597-605.

8 Meador KJ, Baker GA, Browning N, et al. Fetal antiepileptic drug exposure and cognitive outcomes at age 6 years (NEAD study): a prospective observational study. Lancet Neurol 2013;12:244-52.

9 Cummings C, Stewart M, Stevenson M, et al. Neurodevelopment of children exposed in utero to lamotrigine, sodium valproate and carbamazepine. Arch Dis Child 2011;96:643-7.

10 Thomas SV, Ajaykumar B, Sindhu K, et al. Motor and mental development of infants exposed to antiepileptic drugs in utero. Epilepsy Behav 2008;13:229-36.

11 Bromley RL, Mawer G, Love J, et al. Liverpool and Manchester Neurodevelopment Group [LMNDG]. Early cognitive development in children born to women with epilepsy: a prospective report. Epilepsia 2010;51:2058-65.

12 Bromley RL, Mawer G, Clayton-Smith J, et al. Autism spectrum disorders following in utero exposure to antiepileptic drugs. Neurology 2008;71:1923-4.

13 Christensen J, Grønborg TK, Sørensen MJ, et al. Prenatal valproate exposure and risk of autism spectrum disorders and childhood autism. JAMA 2013;309:1696-703.

14 Cohen MJ, Meador KJ, Browning N, et al. Fetal antiepileptic drug exposure: adaptive and emotional/behavioral functioning at age 6years. Epilepsy Behav 2013;29:308-15.

15 Choi CS, Gonzales EL, Kim KC, et al. The transgenerational inheritance of autism-like phenotypes in mice exposed to valproic acid during pregnancy. Sci Rep 2016;6:36250.

16 Marson AG, Al-Kharusi AM, Alwaidh M, et al. The SANAD study of effectiveness of valproate, lamotrigine, or topiramate for generalised and unclassifiable epilepsy: an unblinded randomised controlled trial. Lancet 2007;369:1016-26. 